- There is a lack of a totally unified approach in the management of patients with a history of radiotherapy for head and neck malignancy requiring dental extractions.

- Hyperbaric oxygen is a modality that needs to be explored as a prophylactic measure, in the patients with previous radiotherapy in need for dental extractions or minor oral surgical procedures, for its effectiveness to be fully known.

- There is clear evidence of antibiotic oversubscribing. Oral and maxillofacial surgeons would prescribe antibiotics irrespective of the risk for subsequent osteoradionecrosis.

\title{
A survey of antibiotic prescribing by maxillofacial consultants for dental extractions following radiotherapy to the oral cavity
}

\author{
A. N. Kanatas, ${ }^{1}$ S. N. Rogers ${ }^{2}$ and M. V. Martin ${ }^{3}$
}

\begin{abstract}
Objectives To analyse the antibiotic prescribing trends for exodontia and minor oral surgery in patients with a history of radiotherapy. Also, to evaluate the use of hyperbaric oxygen as a separate modality in the prevention of osteoradionecrosis.
\end{abstract}

Design A survey of antibiotic prescribing involving the analysis of a questionnaire which included the management of three patients with a history of head and neck malignancy.

Method The heads of the departments in each unit in the UK were sent a closed-response questionnaire. Antibiotic prescribing was assessed in three case scenarios. Case one referred to a patient that had surgery alone as part of the management of head and neck cancer, who needed the surgical removal of second molar roots. Case two referred to a patient that had surgery and adjuvant radiotherapy as part of the management of head and neck cancer, and needed the same procedure as in case one. Case three included a patient with a history of surgery and adjuvant radiotherapy, who required the extraction of three mobile and periodontally-involved lower incisors. Consultants were also asked about the use of hyperbaric oxygen in the patients who had radiotherapy as part of their treatment strategy.

Results A total of 109 questionnaires were sent to all the Maxillofacial Units in the UK. The response rate was $73 \%$. In patients with a history of radical surgery alone for the management of head and neck cancer, 20\% of the surgeons advocated pre-operative antibiotics for the surgical removal of lower posterior teeth. In contrast, in the patient with a history of adjuvant radiotherapy $86 \%$ supported pre-operative antimicrobial use for the surgical removal of the same teeth. In the extraction of mobile and periodontally involved lower incisors in the previously irradiated patient, $63 \%$ of the clinicians supported pre-extraction antibiotics. Postoperative antibiotics were advocated in 52\% in the first case, $89 \%$ in the second case and $71 \%$ in the third case. $34 \%$ of the clinicians advocated hyperbaric oxygen for surgical removal of posterior teeth and 15\% for the extraction of mobile anterior teeth.

${ }^{1}$ Hospital Practitioner, Liverpool University Dental Hospital, Pembroke Place, Liverpool L3 5PS $2^{*}$ Consultant Oral and Maxillofacial Surgeon, Regional Centre for Maxillofacial Surgery, University Hospital Aintree, Lower Lane, Fazakerly, Liverpool L9 1AE

3Senior Lecturer in Oral Microbiology, Department of Clinical Dental Sciences, University of Liverpool School of Dentistry, Daulby Street, L69 3GN

${ }^{*}$ Correspondence to: S. N. Rogers

email:snrogers@globalnet.co.uk

\section{Refereed paper}

Received 23.04.01; Accepted 23.08.01

$\odot$ British Dental Journal 2002; 192: 157-160
Conclusions The use of peri-extraction antibiotics was favoured among oral and maxillofacial surgeons. There was little enthusiasm towards the use of hyperbaric oxygen as a prophylactic measure, alone or in conjunction with an antimicrobial regime. Control randomised trials that will measure the effectiveness of hyperbaric oxygen and the appropriate use of antibiotics for prophylaxis in pre- and post-operative regimes are necessary to evaluate the use of these modalities.

Patients who have radiotherapy, as part of their management of head and neck cancer are at risk of developing jaw osteoradionecrosis (ORN) following dental extractions. ${ }^{1,2}$ Teeth should be kept wherever possible as chewing function has an important bearing on the quality of life., ${ }^{3,4}$ The presence of teeth and their later extraction places the patient at risk of developing one of the most severe complications of radiotherapy, that of ORN. Traditionally ORN has been defined as the exposed irradiated bone that fails to heal over a period of 3 months. ${ }^{5}$ It is a chronic condition, it is not the same as a dry socket, and the risk of developing it does not diminish with time. ${ }^{6,7}$ The accepted cause is a progressive endarteritis with a decrease in the microcirculation. ${ }^{8}$ The periosteum undergoes fibrosis, osteoblasts and osteocytes are destroyed and the marrow spaces in the bone become filled with fibrous tissue. The major risk factors for ORN are extractions or biopsies that overly bone. Although ORN can occur spontaneously, these procedures place the patient in great danger. ${ }^{1}$

Predisposing factors for ORN include anatomic location of the tumour, total radiation dose, mode of radiation delivery and dental status. ${ }^{9}$ Superimposed on the rate of developing ORN, extractions have been implicated more often than any other factor. ${ }^{1}$ ORN is more likely to arise after the removal of mandibular molars located directly in the treatment field. The chance of developing ORN after dental extraction ranges from 0 to $65 \%$, but averages 5.8\% when data from different studies are analysed..$^{10}$ The importance of dental extractions is given additional significance because mandibular resection is more frequently required if ORN occurs following extractions. ${ }^{11}$ The healing capacity of head and neck cancer patients can be compromised and Kluth et al. ${ }^{12}$ emphasised smoking, alcohol and nutritional status as other risk co-factors.

Recommendations have been made in surgical practice in an attempt to reduce the risk of developing ORN. These can be broadly classified into preventative oral care, ${ }^{13-16}$ adjuvant therapies such 
as use of hyperbaric oxygen and ultrasound, ${ }^{17,18}$ modified surgical techniques ${ }^{6,18,19}$ and a combination of pre- and post-operative antimicrobial protocols. ${ }^{6,10,20-24}$

As ORN is a chronic condition of bone, some authors have suggested antibiotics that have an affinity for bone and advocate a prolonged course of treatment. ${ }^{19,25-27}$ The prevention of ORN following radiotherapy presents a challenge. Many authors of articles on ORN either failed to mention antibiotics at all, or made only a brief comment about their general application. 1,5,8,11,12,28-30

The purpose of this investigation was to determine prescribing for exodontia and minor oral surgery following radiotherapy by surveying current opinion amongst consultant oral and maxillofacial surgeons.

\section{MATERIALS AND METHODS}

The heads of the departments in each oral and maxillofacial surgery unit in the UK were sent a questionnaire. Consultants were asked about antibiotic prescribing in three carefully constructed case scenarios. Case one referred to a patient that had surgery alone as part of the management of head and neck cancer, who needed the surgical removal of second molar roots. Case two was referred to a patient that had combination therapy, which included primary surgery and adjuvant radiotherapy as part of the management of head and neck cancer, in need of the same procedure as in case one. Finally, case three included a patient with a history of primary surgery with adjuvant radiotherapy, who required the extraction of three mobile and periodontally-involved lower incisors. The first case allowed comparison with the two other cases, which had adjuvant radiotherapy. Cases one and two provided information about minor oral surgery procedures when case three gave an insight into the management of simple extractions. Consultants were also asked about the use of hyperbaric oxygen (HBO) in the patients who had radiotherapy as part of their treatment strategy. Copies of the questionnaire are available from the lead author.

There are 129 oral and maxillofacial units in the UK and because some maxillofacial consultants covered more than one unit the survey size was 109 . Their responses were examined and their significance assessed using the $\chi^{2}$ test.

\section{RESULTS}

A total of 109 questionnaires were sent to all the maxillofacial units in the UK; 79 questionnaires were returned (73\% response rate). Three questionnaires were not completed and 11 of the 79 were returned incorrectly marked. The results of the remainder $82 \%$ were presented below in Table 1 . Cancer patients were treated by $72 / 79$ units.

Responding to case one in which the treatment had been restricted to surgical intervention alone 13/65 (20\%) of the surgeons advocated pre-operative antibiotics. In contrast, in the second case scenario 56/65 (86\%) supported pre-operative antimicrobial use. In the third case scenario which involved extraction of mobile and periodontally involved lower incisors 41/65 (63\%) clinicians supported pre-extraction antibiotics. The route of administration at induction was intravenous 12/13 in case one, 55/56 in case two, 41/41 in case three. One of the clinicians advocated suppository antibiotics for the relevant patient groups in the first and second cases.

Post-operative antibiotics were advocated in 34/65 (52\%) in case one, in 58/65 (89\%) in case two and in 46/65 (71\%) in case three. In the previously irradiated patient that required the surgical removal of posterior teeth six of the responders did not support a course of post-op antibiotics, but suggested one dose of post-operative intravenous antibiotics. Analysing the antibiotics chosen (Figs 1 and 2) in the second and third case scenario using a $\chi^{2}$ test, the probabilities of the choices being a random sample was less than $0.001(\mathrm{P}<0.001)$. The most popular choice of antibiotic overall was amoxicillin in patients not allergic to penicillins and clindamycin in those with allergy. Of the consultants prescribing post-operative antibiotics for the last two case scenarios, 39/44 (89\%) used the same antibiotic on each occasion. The range in duration of post-extraction antibiotics varied from 3 to 28 days, although one consultant prescribed antibiotics for cases two and three until the healing was completed.

Considering the use of HBO, 22/65 (34\%) of the clinicians advocated HBO in case two, in contrast to case three where 10/65 (15\%) were in favour of such modality.

\section{DISCUSSION}

Radiotherapy can positively affect the patient's survival and is considered a potent therapeutic tool against malignancy. Occasionally the spectrum of complications includes osteoradionecrosis, a distressing condition that can influence the rate of tissue recovery, as well as the patient's prosthetic rehabilitation. The incidence of ORN varies considerably in different studies ranging from $2 \%$ to $85 \% .^{10,22,24,28}$ In general, the risk is significantly higher for the dentate than for the edentulous patient.

When an extraction is contemplated in this patient group, there is no consensus on how it is to be performed. There is no doubt that the general dental practitioner makes an important contribution to the head and neck cancer team by helping to maintain an optimal oral hygiene. However, for the procedure itself suggestions included a radical alveolectomy ${ }^{6}$ so that tension-free watertight mucosal coverage of bone can be achieved. In one article ${ }^{18}$ alveolectomy was recommended together with nasogastric feeding for 8 days.

The literature generally supports the use of peri-extraction antibiotics ${ }^{19,25,26,27,31,32}$ for patients at risk of ORN, however there is an evident lack of detail concerning the type, dose and timing of antibiotic prophylaxis. Evidence is lacking upon which to base unequivocal guidelines as reflected by the diversity of practice reported in this survey. Pre-extraction antibiotics on the patient that had previous radiotherapy were clearly favoured when compared with the patient that had surgery alone. The use of prophylactic amoxicillin was the first choice in the non-allergic patient, followed by augmentin and the combination of amoxicillin with metronidazole. In the patient with a penicillin allergy, a preference to clindamycin followed by metronidazole and the combination of a cephalosporin with metronidazole has been recorded (Figs 1 and 2).

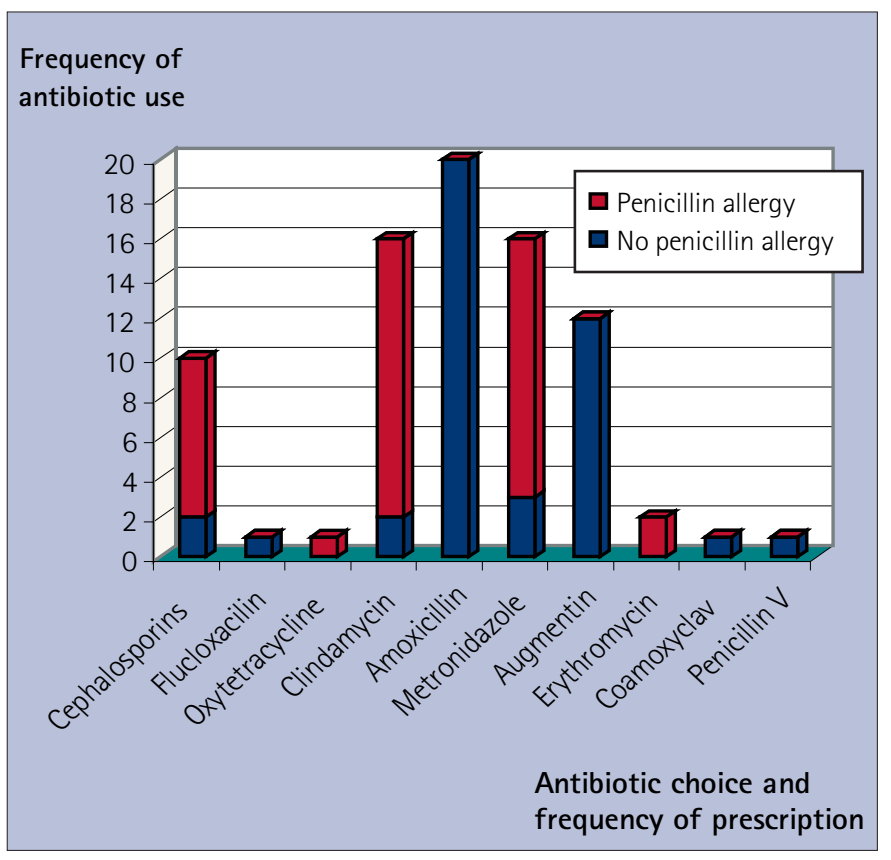

Fig. 1 Post-operative antibiotics given in the previously irradiated patient with and without penicillin allergy 


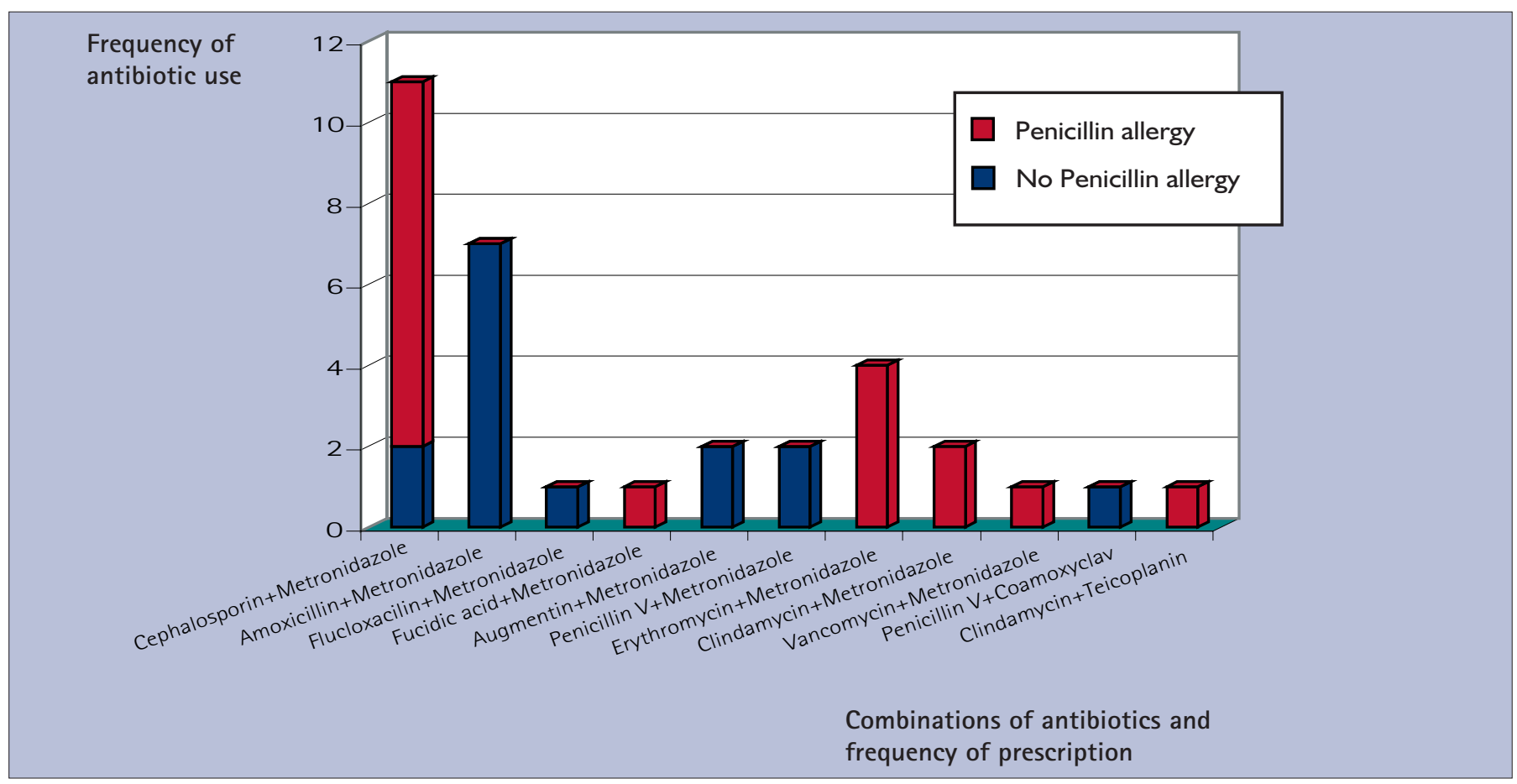

Fig. 2 Combinations of antibiotics given post-operatively in the previously irradiated patient with and without penicillin allergy

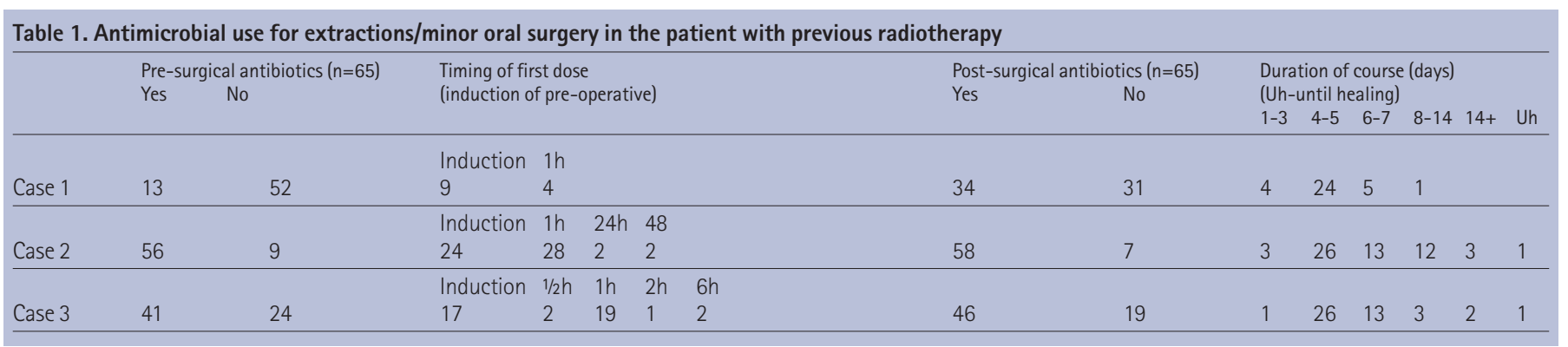

Although the use of amoxicillin, metronidazole and clindamycin are choices which conform with recent recommendations $^{31}$ the duration of the course given varied from 3 to 28 days and on one occasion until healing had been completed (Table 1). Such action exposes the absence of a definitive oral health protocol for patients at risk of ORN. There is also clear evidence of overprescribing in patients at risk of ORN. Inappropriate use of antibiotics leads to the development of antibiotic resistance. ${ }^{33}$ In addition, the presentation of a high dose of an antibiotic agent can result in a fatal adverse host response. Antimicrobials may also cause damage to blood vessels and have adverse effects on the respiratory and cardiovascular systems. ${ }^{34}$ The severity of ORN and the required aggressive management has been clearly reflected after direct comparison of the intended actions in cases one and two of the questionnaire.

The majority of oral and maxillofacial surgeons would prescribe antibiotics irrespective of risk for subsequent ORN as it appeared when analysing data from cases two and three. No significant difference in the antibiotic choice was obtained, with 39/44 adopting the same measures. Clearly for cases two and three the consensus supported antibiotic use at induction or 1 hour pre-operatively. There were no significant differences between the intravenous and the oral route of antibiotic administration.

Although the clinical application of hyperbaric oxygen is regarded as an additionally helpful treatment modality in various medical specialties, ${ }^{35}$ this was not the case in the data collected. Surgeons were reluctant to substitute the use of antibi- otics with HBO. The relatively low use of HBO is at first glance surprising considering the very low ORN reported by Marx in the only available randomised trial, comparing HBO alone versus penicillin in the management of extractions. Marx et al. ${ }^{17}$ reported an incidence of ORN in the HBO group of 5.4\% versus $29.9 \%$ in the antibiotic group. HBO may not be a popular option for reasons such as geographical location, costs, and lack of perceived necessity. Taking into account anecdotal comments on the last section of the questionnaire, availability of the specific equipment, as well as the resources available in the clinical hyperbaric centres currently adversely affects the use of HBO prophylactically. It appears that this choice is reserved for the management of established cases of ORN and almost always in combination with substantial doses of intravenous antibiotics.

Analysis of the data represents only an indication of how extractions are managed in the irradiated patient. Carefully designed randomised trials are needed for establishing the relative importance of the type, dose, timing and duration of antibiotics. In the absence of any such trials, it would seem reasonable to suggest that for patients who require extractions or other minor oral surgery following radiotherapy are best managed in an oncology centre. Prophylactic antibiotics should be considered with either amoxicillin or metronidazole or clindamycin perioperatively and possibly for up to five days postoperatively. When available and in cases where radiation dose to the mandible has been greater than 50 gray, prophylactic HBO should be considered. ${ }^{30,36}$ 
1. Widmark G, Sagne S, Heikel P. Osteoradionecrosis of the jaws. Int J Oral Maxillofac Surg 1989; 18: 302-306.

2. Benjelloum A, Boulaich M, Alaoui Rachidi F et al. Osteoradionecrosis of the jaws. Apropos of 7 cases. Rev Stomatol Chir Maxillofac 1998: 99: 192-198.

3. Allison P J, Locker D, Feine J S. The relationship between dental status and healthrelated quality of life in upper aerodigestive tract cancer. Oral Oncol 1999; 35: 138143.

4. Rogers S N, McNally D, Mahmood M, Chan M, Mahmood M, Humphris G M. The psychological response of the edentulous patient following primary surgery for oral cancer: A cross-sectional study. J Prosthet Dent 1999; 82: 317-321.

5. Hao S-P, Chen H C, Wei F-C. Systematic management of osteoradionecrosis in the head and neck. Laryngoscope 1999; 109: 1324-1328.

6. Beumer J, Seto B. Dental extractions in irradiated patient. Spec Care Dentist 1981; 1: 166-173.

7. Marx R E, Johnson R P. Studies in the radiobiology of osteoradionecrosis and their clinical significance. Oral Surg Oral Med Oral Path 1987; 64:379-390.

8. Lambert P M, Intriene N, Eichstaedt R. Clinical controversies in oral and maxillofacial surgery: Part one. Management of dental extractions in irradiated jaws: a protocol with hyperbaric oxygen therapy. J Oral Maxillofac Surg 1997; 55: 268-274.

9. Curi M M, Dib L L. Osteoradionecrosis of the jaws: a retrospective study of the background factors and treatment in 104 cases. J Oral Maxillofac Surg 1997; 55: 540-544.

10. Clayman L. Clinical controversies in oral and maxillofacial surgery: Part two Management of dental extractions in irradiated jaws: a protocol without hyperbaric oxygen therapy. J Oral Maxillofac Surg 1997; 55: 275-281.

11. Beumer J, Harrison R, Saunders B et al. Osteoradionecrosis: predisposing factors and outcomes of therapy. Head Neck Surg 1984; 6: 819-827.

12. Kluth EV, Rani Jain P, Stuchell R N, Frich J C. A study of factors contributing to the development of osteoradionecrosis of the jaws. J Prosthet Dent 1998; 59: 194-201.

13. Nicholls $C$, Ilankovan $V$. An audit of oral and dental health regimes practised in the management of oropharyngeal cancer. Br J Oral Maxillofac Surg 1998; 36: 63-66.

14. Joyston-Bechal S. Prevention of dental diseases following radiotherapy and chemotherapy. Int Dent J 1992; 42: 47-53.

15. Jasma J, Vissink A, Bouma J, Vermey A, Panders A, Gravenmade J. A survey of prevention and treatment regimes for oral sequelae resulting from head and neck radiotherapy used in Dutch radiotherapy institutes. Int J Radiat Oncol Biol Phys 1992; 24: 359-367.

16. Jasma J, Vissink A, Spijkervet F et al. Protocol for the prevention and treatment of oral sequelae resulting from head and neck radiation therapy. Cancer 1992; 70: 2171-2179.

17. Marx R E, Johnson R P, Kline S N. Prevention of osteoradionecrosis: A randomised prospective clinical trial of hyperbaric oxygen versus penicillin. J Am Dent Assoc 1985; $111: 49-54$
18. Horiot J $\mathrm{C}$ et al. Systematic dental management in head and neck irradiation. Int J Radiat Oncol Biol Phys 1981; 7: 1025-1029.

19. Epstein J, Van der Meij E, McKenzie M. et al. Postradiation osteonecrosis of the mandible. A long term follow up study. Oral Surg Oral Med Oral Pathol Oral Radiol Endod 1997; 83: 657-662.

20. Daly TE, Drane J B,MacComb W S. Management of problems of the teeth and jaws in patients undergoing irradiation. Am J Surg 1972; 124: 539-542.

21. Morton M E. Osteoradionecrosis: a study of the incidence in the North West of England. Br J Oral Maxillofac Surg 1986; 24: 323-331.

22. Morton ME, Simpson W. The management of osteoradionecrosis of the jaws. Br J Orat Maxillofac Surg 1986; 24: 332-334.

23. O'Sullivan B R, Oatis G W, Grisius R J. Osteoradionecrosis: its prevention. Clin Prevent Dent 1982; 4: 8-12.

24. Patel P, Raybould T, Maruyama Y. Osteoradionecrosis of the jaw bones at the University of Kentucky Medical Center. J Ky Med Assoc 1989; 87: 327-331.

25. Coffin $F$. The incidence and management of osteoradionecrosis of the jaws following head and neck radiotherapy. BrJ Radio/ 1983; 56: 851-857.

26. Haber-Cohen A, Debuski S. Management of oral and maxillofacial surgery procedures in irradiated patients. Dent Clin North Am 1990; 34: 71-77.

27. Singh N, Scully C, Joyston-Bechal S. Oral complications of cancer therapies: prevention and management. Clin Oncol 1996: 8: 15-24.

28. Toljanic J A, Ali M, Haraf D J, Vokes E E, Moran W J, Graham L. Osteoradionecrosis of the jaws as a risk factor in radiotherapy: a report of an eight-year retrospective review. Oncol Rep 1998; 5: 345-349.

29. Epstein J, Van der Meij E, McKenzie M et al. A study of factors contributing to the development of osteoradionecrosis of the jaws. Prosthet Dent 1988; 59: 194-201.

30. Vudiniabola S, Pirone C, Williamson J, Goss A N. Hyperbaric oxygen in the prevention of osteoradionecrosis of the jaws. Aust Dent J 1999; 44: 243-247.

31. Tong A C, Leung A C, Cheng J C, Sham J. Incidence of complicated healing and osteoradionecrosis following tooth extraction in patients receiving radiotherapy for treatment of oropharyngeal carcinoma. Aust Dent J 1999; 44: 187-194.

32. Martin M V, Longman L P, Palmer N A O. Adult antimicrobial prescribing in primary dental care for general dental practitioners. London: Faculty of General Dental Practitioners (UK), 2000.

33. Smith A, Bagg J. An update on antimicrobial chemotherapy: 3. Antimicrobial resistance and the oral cavity. Dent Update 1998; 25: 230-234.

34. Schanker L. Drug absorption. In La Du B N, Mandel H G, Way E L(Ed). Fundamentals of drug metabolism and drug disposition. Baltimore: Williams and Wilkins, 1971.

35. Wattel F, Mathieu D, Neviere R. Indications for hyperbaric oxygen therapy Organisation of the treatment unit. Training of personnel. Bull Acad Natl Med 1996; 180: 949-963.

36. Murray C G, Daly TE, Zimmerman S 0. The relationship between dental disease and radiation necrosis of the mandible. Oral Surg Med Oral Pathol 1980; 49: 99-104. 